\title{
Lightweight Hardware Transactional Memory Profiling
}

\author{
Qingsen Wang, Pengfei Su \\ College of William \& Mary \\ \{qwang06,psu\}@email.wm.edu
}

\author{
Milind Chabbi \\ Scalable Machines Research \\ milind@ScalableMachines.org
}

\author{
Xu Liu \\ College of William \& Mary \\ xl10@cs.wm.edu
}

\section{Artifact Appendix}

\subsection{Abstract}

We have prepared a three-part artifact evaluation:

Overhead of TXSAMPLER: We reproduce the overhead of execution time as shown in Figure 5 in the paper.

Speedup after optimization: We reproduce the speedup of optimized applications as shown in Table 2 in the paper.

Profile Analysis We run TXSAMPLER on the case studies discussed in Section 8 and produce profiles databases. One can view the generated profiles with HPCViewer to navigate the performance problems and optimization opportunities as discussed in the paper.

\subsection{Artifact check-list (meta-information)}

- Program: TxSAmpler

- Compilation: gec -O3

- Data set: RMS-TM, parboil, parsec-2.1, parsec-3.0, stamp0.9.10, bart, Lee, splash2

- Run-time environment: Linux $>=\mathbf{3 . 1 0}$

- Hardware: Intel Xeon Haswell and its successors with Hardware Transactional Memory supported

- Execution: python/bash script

- Output: Figures, text files and TxSampler Profiles

- How much disk space required (approximately)?: 100GB

- Publicly available?: Yes

\subsection{Description}

\subsubsection{How delivered}

All the source codes and inputs of TXSAMPLER and HTM programs are delivered via github and Google drive.

\subsubsection{Hardware dependencies}

The hardware should be Intel Xeon Haswell and its successors with Transactional Synchronization Extensions (TSX) supported.

\subsubsection{Software dependencies}

We need Docker CE and Java SE. We have tested TxSAMPLER on Linux 3.10.0.

\subsubsection{Data sets}

RMS-TM, parboil, parsec-2.1, parsec-3.0, stamp-0.9.10, bart, Lee, splash2.

PPoPP '19, February 16-20, 2019, Washington, DC, USA

2019. ACM ISBN 978-1-4503-6225-2/19/02 .. \$15.00

https://doi.org/10.1145/3293883.3295728

\subsection{Installation}

To avoid the complexity of installing all the dependencies, we have prepared a docker file so that users can build their docker image and perform all the experiments inside the docker. Please refer to https://github.com/jqswang/txsampler-ae for detailed instructions.

\subsection{Experiment workflow}

Follow the instructions shown in https://github.com/jqswang/txsamplerae to build and run the docker image. Once inside the container, download the benchmark suite and input by the following command, and then set the environmental variables.

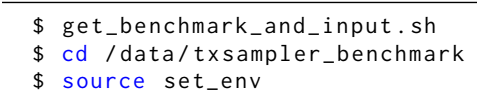

Edit /data/txsampler_benchmark/run. conf to modify the number of threads and the CPU affinity setting instructed in the file comment.

The /data directory inside the container is mapped to . /mydata directory from the host, which can be used to transfer files between the container and the host.

\subsubsection{Overhead of TXSAMPLER}

Use following command to generate the figure of runtime overhead similar to Figure 5.

1 \$ measure_overhead.py all

The generated figure is under the current directory.

\subsubsection{Speedup after optimization}

Issue the following command to measure the speedup of optimized applications as shown in Table 2.

1 \$ measure_speedup.py all

The result is shown in the standard output.

\subsubsection{Profile analysis}

Issue the command to produce profile databases:

\$ generate_profile.py -1 \# list available applications

\$ generate_profile.py [application name]

One can use HPCViewer to open the database directory and check the result. HPCViewer prebuilt on different platforms is available at http://hpctoolkit.org/download/hpcviewer/. 


\subsection{Evaluation and expected result}

Depending on the hardware and the configuration of thread number and core binding, the result may be different from the one presented in the paper.

\subsubsection{Overhead of TXSAMPLER}

The generated figure is similar to Figure 5 . The overhead of a specific application should be between $0 \sim 20 \%$ and the geo-mean is less than $10 \%$.

\subsubsection{Speedup after optimization}

In the speedup analysis, the speedup number will be shown in the terminal which is like Table 2. The speedup should be nontrivial but the exact speedup number is sensitive to the configuration.

\subsubsection{Profile analysis}

One can follow Figure 1 to find optimization opportunities. Section 8.1 provides detailed instructions. The metric pane shows many metrics, from which we can derive new metrics. For example, the capcity abort in Figure 9 is the sum of capacity abort read and capacity abort write. For backup, we have also provided a profile database of dedup (see https://github.com/jqswang/txsamplerae), which can be directly opened by HPCViewer. 